\title{
Microlever with combined integrated sensor/actuator functions for scanning force microscopy
}

\author{
J. Brugger ${ }^{a}$, N. Blanc ${ }^{a}$, Ph. Renaud ${ }^{b}$ and N.F. de Rooij ${ }^{a}$ \\ "Institute of Microtechnology (IMT), University of Neuchâtel, Bneguet 2, 2000 Neuchâtel (Switzerland) \\ 'Swiss Center for Electronics and Microtechnology (CSEM) Inc., Maladière 71, 2000 Neuchâtel (Switzerland)
}

(Received August 13, 1993; accepted December 2, 1993)

\begin{abstract}
A novel silicon microfabricated sensor head for the scanning force microscope (SFM) is presented. The force sensor consists of a cantilever and an adjacent counter-electrode forming the two plates of a capacitor. Forceinduced cantilever deflections are monitored by capacitive detection. Typical lever dimensions of $800 \mu \mathrm{m} \times 40$ $\mu \mathrm{m}$ and a gap of $3 \mu \mathrm{m}$ yield an active sensing capacitance $C=0.1 \mathrm{pF}$. The expected sensitivity in terms of vertical cantilever motion is $\Delta C / \Delta z=10 \mathrm{fF} / \mu \mathrm{m}$. In addition to the sensing capability, the microlever can also be $z$-actuated by applying controlled voltages. This allows both the tip-to-sample distance and the cantilever/system compliance to be adjusted. Expressions are derived for the amplitude of cantilever deflections under electrostatic actuation in the static and dynamic modes as pertinent to applications of SFM in the contact and non-contact modes. The microlever is fabricated using silicon bulk- and surface-micromachining techniques including fusion bonding and sacrificial layer etching. First measurements of the static and dynamic deflections of cantilevers are analysed and show promising results. The reported device basically represents a module of an SFM microsystem with integrated cantilever deflection sensor and adjustment capability.
\end{abstract}

\section{Introduction}

In scanning force microscopy local surface forces acting between a sample and a sharp tip are probed via the deflection of a tiny cantilever beam [1]. The cantilever motions, usually in the nanometre and subnanometre range, are sensed with different measuring techniques including assembled optical, tunnelling or capacitive set-ups [2]. All these detection principles require external physical components to be aligned, somewhat complicating the system design.

In consideration of an integrally designed scanning force microscopy (SFM) sensor head, a piezoresistive cantilever [3] and a piezoelectric lever [4] have been operated successfully. Furthermore, a bimorph structure (metal/piezoelectric zinc-oxide) providing integrated three-dimensional tip actuation has also been presented [5]. Such sensing and actuated cantilevers are of great interest for miniaturized scanning probe systems, not least for industrial applications such as IC surface profiling. In particular, they enable the scanning of the tip versus the fixed sample while having the sensor incorporated in the probe, thus substantially simplifying the design of a stand-alone SFM microsystem.

The capacitive method for cantilever deflection measurements has proven to be an interesting alternative to other methods [6-9]. The fabrication and operation of such a miniaturized capacitive probe are inherently critical. Strict instrumental requirements have to be ensured in order to control and minimize stray capacitance effects for a high-resolution system.

Accurate machining and alignment of the capacitor plates and gap are crucial for successful operation. Silicon micromachining is a promising technique to batch-fabricate reproducibly such devices with high accuracy. In this paper we present a fully micromachined capacitive microlever for the SFM. The device has the interesting asset of incorporating both a cantileverdeflection sensing and adjustment capability in a single probe. While profiling a sample surface in the quasistatic mode, minute cantilever deflections induce a change in the capacitance gap between two silicon beams, which can be measured via interface electronics. Alternatively, when vibrating and scanning the microlever in a non-contact dynamic mode, the frequency shift due to force gradients can be measured. Silicon is well suited for resonant methods due to its inherently high $Q$-factor [10].

The electrostatic actuation capability by applying controlled voltages allows for an induced cantilever bending, thus permitting the tip-sample fine approach and eventually an autonomous feedback system. Fur- 
thermore, the adjustment/tuning possibility of the lever's mechanical characteristics, and thus of the contact pressure between tip and sample, gives access to new fields of SFM applications. The reported device basically represents a microfabricated component of a standalone SFM system. We first present the design and the modelling of the capacitive microlever. In particular the stability and snapping criterion of the microelectromechanical device are considered. The microfabrication is then described and first prototypes arc characterized.

\section{Design and modelling}

The micromachined monocrystalline silicon SFM microlever (Figs. 1 and 2) consists of a cantilever carrying a sharp tip and an adjacent countcr-elcctrode attached to the substrate holder, which form a parallel-plate airgap capacitor. The mechanical properties of the cantilever are given by the material constants and the geometrical dimensions length $l$, width $w$ and thickness $t$. In particular, the spring constant $k$ and the resonance frequency $\omega_{0}$ are given by [11]

$k=3 \frac{E I}{l^{3}}$

and

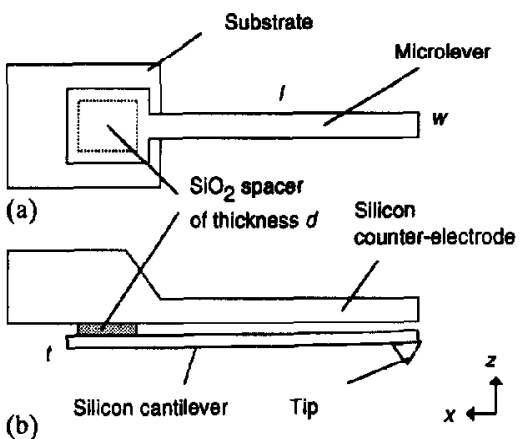

Fig. 1. Scbeme of the capacitive microlever: (a) top view denoting length $l$ and width $w$; (b) side view showing the $\mathrm{SiO}_{2}$ spacer and the air-gap capacitor.

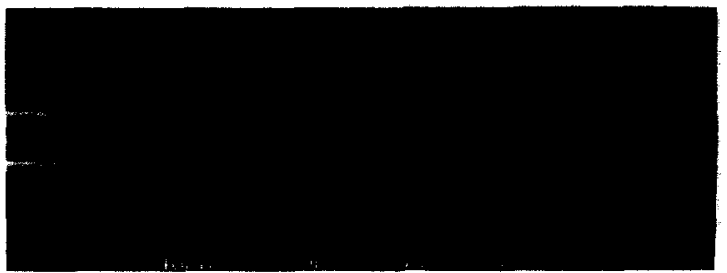

Fig. 2. Scanning electron micrograph of the capacitive micromachined monocrystalline silicon SFM cantilever with tip and the adjacent counter-electrode.
$\omega_{0}=\frac{1.875^{2}}{l^{2}}\left(\frac{E I}{w t \rho}\right)^{1 / 2}=\left(\frac{k}{0.24 m}\right)^{1 / 2}$

with $I=w t^{3} / 12$ the moment of inertia, $E=1.7 \times 10^{11}$ $\mathrm{N} / \mathrm{m}^{2}$ Young's modulus, $\rho=2300 \mathrm{~kg} / \mathrm{m}^{3}$ the density of the silicon and $m=\rho w t l$ the mass of the cantilever. One of our devices with typical dimensions $l=800 \mu \mathrm{m}$, $w=40 \mu \mathrm{m}$ and $t=5 \mu \mathrm{m}$ yields $k \approx 0.5 \mathrm{~N} / \mathrm{m}$ and $f=$ $\omega_{0} / 2 \pi \approx 10 \mathrm{kHz}$. Equation (2) has the form of a simple harmonic oscillator $\omega_{0}=\left(k / m_{\mathrm{eff}}\right)^{1 / 2}$ with $m_{\mathrm{eff}}=0.24 \mathrm{~m}[11]$. The active sensing capacitance is given by $C=\epsilon_{0} A / d$ with $A=w l, d=3 \mu \mathrm{m}$ for the gap and $\epsilon_{0}=8.84 \times 10^{-12}$ A s $/ V \mathrm{~m}$ and yields $C \approx 0.1 \mathrm{pF}$ (see Table 1). The parallel capacitance of the anchoring pad of silicon dioxide amounts to about $1 \mathrm{pF}$. Due to the simply clamped cantilever, only part of the capacitor area $A$ contributes effectively to the deflection sensing and actuation. As shown below, a more accurate model is obtained by redefining an effective area $A_{\text {eff }}=(3 / 8) A$.

\section{Cantilever deflection sensor}

When raster scanning the tip across a sample, local surface forces will induce a minute defiection $z$ of the cantilever beam. The elastic curve due to a concentrated load at its end can be expressed by [12]

$z(x)=\frac{z_{0}}{2 l^{3}}\left(x^{3}-3 l^{2} x+2 l^{3}\right)$

where $z_{0}$ is the deflection at the free end. This cantilever motion changes the air-gap capacitance $C$ computed according to

$C\left(z_{0}\right)=\int_{0}^{l} \frac{\epsilon_{0} w}{d-z(x)} d x$

In Fig. 3 we have calculated the sensitivity $\Delta C / \Delta \mathrm{z}_{0}$ by numerical differentiation of eqn. (4) for a typical cantilever and various gap values $(d=1,2,3, \mu \mathrm{m})$. The sensitivity at zero deflection $\left(z_{0}=0\right)$ can be expressed in an analytical form as

$\frac{\Delta C}{\Delta z_{0}}\left(z_{0}=0\right)=\epsilon_{0} \frac{3 w l}{8 d^{2}}$

TABLE 1. Typical microlever dimensions

\begin{tabular}{ll}
\hline Length $l$ & $800 \mu \mathrm{m}$ \\
Width $w$ & $40 \mu \mathrm{m}$ \\
Thickness $t$ & $5 \mu \mathrm{m}$ \\
Spring constant $k$ & $0.5 \mathrm{~N} / \mathrm{m}$ \\
Resonance frequency $f$ & $10 \mathrm{kHz}$ \\
Area $A=l \times w$ & $800 \times 40 \mu \mathrm{m} \times \mu \mathrm{m}$ \\
Gap $d$ & $3 \mu \mathrm{m}$ \\
Active capacitance $C$ & $0.1 \mathrm{pF}$ \\
Sensitivity $\Delta C / \Delta z$ (calculated) & $10 \mathrm{fF} / \mu \mathrm{m}$
\end{tabular}




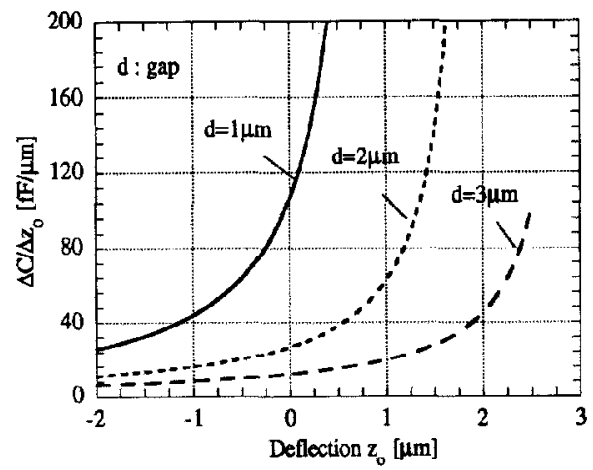

Fig. 3. Calculated sensitivity $\Delta C / \Delta z_{B}$ of the capacitive microlever as a function of $z_{0}$ for different gap values $(d=1-3 \mu \mathrm{m}) ; l=800$ $\mu \mathrm{m}, w=40 \mu \mathrm{m}$, positive and negative deflections are shown.

This corresponds to the sensitivity for a simple parallelplate capacitor of area $A_{\text {eff }}=(3 / 8) A$. For our typical device we obtain $\Delta C / \Delta z_{0} \cong 10 \mathrm{fF} / \mu \mathrm{m}$ (Table 1).

\section{Cantilever z-actuation}

In addition to the deflection-sensing capability, the microlever can also be used for $z$-actuation of the tip by applying controlled voltages on the probe. In the case of a parallel-plate capacitor of area $A$, gap $d$ and applied voltage $V$, the electrostatic force is [13]

$$
\begin{aligned}
F_{\mathrm{el}} & =\frac{1}{2} \epsilon_{\mathrm{o}} \frac{A}{d^{2}} V^{2} \\
& \approx \frac{1}{2} \epsilon_{0} \frac{A}{d^{2}}\left(V_{\mathrm{dc}}^{2}+2 V_{\mathrm{dc}} V_{\mathrm{ac}} \cos \omega t\right)
\end{aligned}
$$

assuming $V=V_{\mathrm{dc}}+V_{\mathrm{ac}} \cos$ wt with $V_{\mathrm{dc}} \gg V_{\mathrm{ac}}$ so that high-order terms do not contribute.

\section{Static deflection $\left(V_{a c}=0\right)$}

In the analysis of the actuation, a mathematical complication comes from the deflection of the cantilever and the non-uniform electrostatic forces present along its length. For small deffections, however, the cantilever senses approximately a constant electrostatic pressure

$$
P_{\mathrm{el}}=\frac{1}{2} \epsilon_{0} \frac{V^{2}}{d^{2}}
$$

which leads to a displacement of the tip given by [12]

$z_{0}=\frac{1}{16} \epsilon_{0} \frac{V^{2}}{d^{2}} \frac{w l^{4}}{E I}$

For a concentrated force

$$
F_{\mathrm{el}}=\frac{1}{2} \epsilon_{\mathrm{o}} \frac{A_{\mathrm{eff}} V^{2}}{d^{2}}
$$

acting at the end of the cantilever, the displacement is $z_{0}=\frac{1}{6} \epsilon_{0} \frac{A_{\text {eff }} V^{2}}{d^{2}} \frac{l^{3}}{E I}$

Comparison of eqns. (7) and (8) shows that the same tip displacement is obtained provided one defines an effective area $A_{\text {eff }}=(3 / 8) \mathrm{Wl}$. In the following the electrostatic force is approximated by a concentrated load at the cantilever end. The overall behaviour of the device is best described by computing the total potential energy $U_{\text {tor }}$ of the system, which is the sum of the strain energy of the deflected cantilever and the electrostatic energy due to the driving voltage $V$ [14], thus

$U_{\mathrm{tot}}=\frac{1}{2} k z_{0}^{2}-\frac{1}{2} \epsilon_{0} \frac{A_{\mathrm{eff}}}{\left(d-z_{0}\right)} V^{2}$

Figure 4 shows the calculated potential energy $U_{\text {tor }}$ for our typical cantilever and for different applied voltages. The curves yield information on the cantilever displacement and stability conditions. The cantilever equilibrium positions, for example, are found by solving $\mathrm{d} U_{\text {tot }} / \mathrm{d} z=0$, yielding $k z_{0}=0.5 \epsilon_{0} A_{\text {eff }} V^{2} /\left(d-z_{0}\right)^{2}$, i.e., equating the spring restoring force to the electrostatic force. These two opposite forces generate a stable position of static equilibrium at the minimum $\left(U_{\min }\right)$ and an unstable equilibrium at the maximum $\left(U_{\max }\right)$ of the potential energy, respectively (curves a and $b$ ). Beyond a threshold voltage $V_{1}$ (curves $c$ and $d$ ) no stable equilibrium position exists $\left(\mathrm{d}^{2} U_{\text {tot }} / \mathrm{d} z^{2}<0\right)$ and the cantilever can no longer withstand the attractive electrostatic force and 'snaps' into the counter-electrode. The snapping distance $z_{1}$ and voltage $V_{1}$ can be calculated by satisfying $\mathrm{d} U_{\text {tot }} / \mathrm{d} z\left(z=z_{\mathrm{t}}\right)=0$ and $\mathrm{d}^{2} U_{\text {tot }} / \mathrm{d} z^{2}\left(z=z_{\mathrm{t}}\right)=0$, which yields [13]

$z_{4}=\frac{d}{3}$ and $V_{1}=\left(\frac{8 d^{3} k}{27 \epsilon_{0} A_{\text {eft }}}\right)^{1 / 2}$

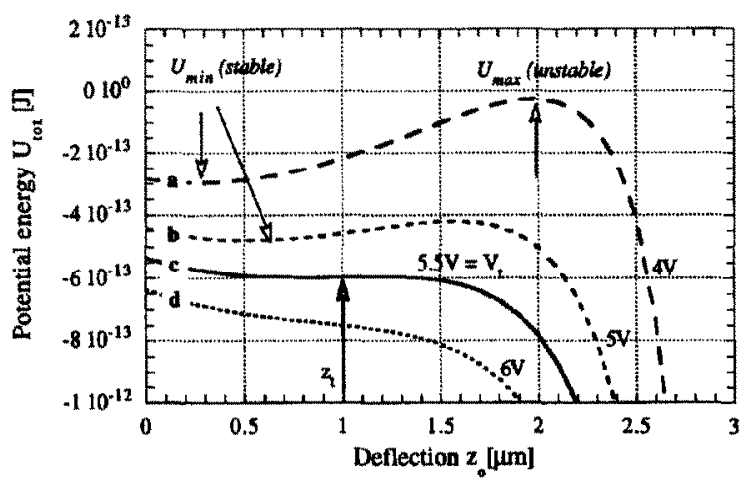

Fig. 4. Calculated total potential energy $U_{\text {tot }}$ as a function of $z_{0}$ for the capacitive microlever showing the equilibrium positions. Threshold voltage $V_{t}=5.5 \mathrm{~V}$ and snapping distance $z_{\mathrm{t}}=1 \mu \mathrm{m}$. 
meaning that the actuation range is limited to one third of the gap $d$. In our case we obtain $z_{\mathrm{t}}=1 \mu \mathrm{m}$ and $V_{\mathrm{t}} \approx 5.5 \mathrm{~V}$.

The effective cantilever compliance $k^{\prime}$ is modified by the attractive forces resulting from the electrostatic field between the cantilever and the counter-electrode and can be calculated with the same model as

$k^{\prime}(V)=\mathrm{d}^{2} U_{\mathrm{tot}} / \mathrm{d} z_{0}^{2}=k-\frac{\epsilon_{0} A_{\text {eff }}}{\left(d-z_{0}\right)^{3}} V^{2}$

leading to a lowering of the resonance frequency $\omega_{0}^{\prime}$ [13]

$\frac{\omega_{0}^{\prime}(V)}{\omega_{0}}=\left(\frac{k^{\prime}}{k}\right)^{1 / 2} \approx 1-\frac{4}{27}\left(\frac{V}{V_{t}}\right)^{2}$

\section{Dynamic deflection}

The forced vibrations of a cantilever due to the driving electrostatic force

$F=\epsilon_{\mathrm{o}} \frac{A_{\mathrm{eff}}}{d^{2}} V_{\mathrm{dc}} V_{\mathrm{ac}} \cos \omega t$

(eqn. (6)) with a viscous damping $-c(\mathrm{~d} z / \mathrm{d} t)$ satisfy the equation of motion

$m_{\mathrm{eff}} \frac{\mathrm{d}^{2} z}{\mathrm{~d} t^{2}}=-k z-c \frac{\mathrm{d} z}{\mathrm{~d} t}+\epsilon_{0} \frac{A_{\mathrm{eff}}}{d^{2}} V_{\mathrm{dc}} V_{\mathrm{ac}} \cos \omega t$

where $m_{\text {eff }}$ is the effective mass of the lever [11]. In the steady state the displacement $z$ from the static equilibrium position is given by the standard resonance equation [15]

$z=\epsilon_{\mathrm{o}} \frac{A_{\mathrm{eff}}}{k d^{2}} V_{\mathrm{dc}} V_{\mathrm{ac}} \beta \cos (\omega t-\Theta)$

in which the magnification factor is

$\beta=\left[\left(1-\omega^{2} / \omega_{0}^{2}\right)^{2}+\left(\omega / Q \omega_{0}\right)^{2}\right]^{-1 / 2}$

and the phase angle is

$\Theta=\tan ^{-1}\left(\frac{\omega / Q \omega_{0}}{1-\omega^{2} / \omega_{0}^{2}}\right)$

with the quality factor $Q=m_{\text {eff }} \omega_{0} / c$.

\section{Device fabrication}

The structure is completely fabricated by means of silicon micromachining techniques as illustrated in Fig 5. The process is based on the fabrication of monocrystalline silicon SFM cantilevers with tips [16] and extended by additional steps including silicon fusion bonding (SFB) and reactive ion etching (RIE). After thermal oxidation to $1.5 \mu \mathrm{m} \mathrm{SiO}_{2}$ of two $280 \mu \mathrm{m}$ thick
Si
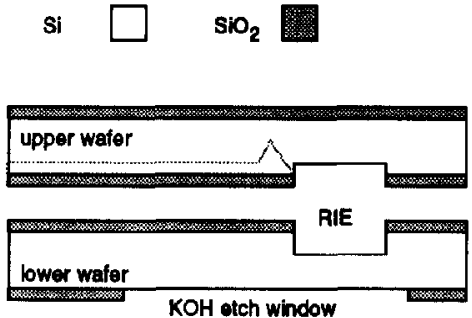

(a)

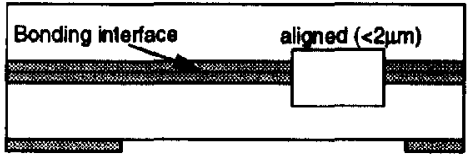

(b)

Bulk otching $(\mathrm{KOH})$

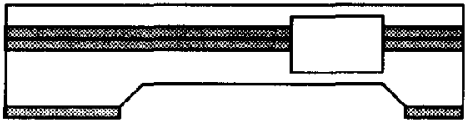

(c)

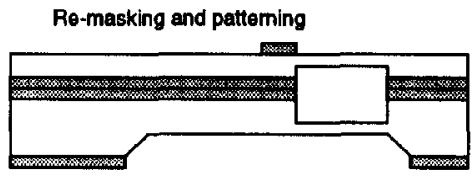

(d)

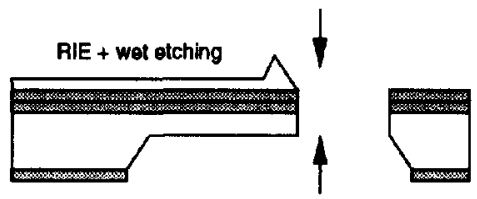

(e)

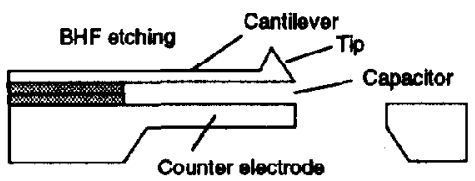

(f)

Fig. 5. Microfabrication of the capacitive microlever (see text).

phosphorus-doped silicon wafers $(\rho \approx 0.03 \Omega \mathrm{cm})$, both wafers are patterned by photolithography and $\mathrm{SiO}_{2}$ etching in buffered hydrofiuoric acid (BHF); the upper wafer with the cantilever shape (see dashed line in Fig. 5(a)), the lower wafer with the counter-electrode and the backside etch windows. Both wafers are RIE processed to form deep trenches of about $5 \mu \mathrm{m}$ for the cantilever and $20 \mu \mathrm{m}$ for the counter-electrode, respectively. After surface cleaning and hydrophilization in $\mathrm{HNO}_{3}$ and $\mathrm{H}_{2} \mathrm{SO}_{4} / \mathrm{H}_{2} \mathrm{O}_{2}$, the two bond partners are aligned together with high precision (accuracy $<2 \mu \mathrm{m}$ ) on a mask aligner and pre-bonded by applying a con- 


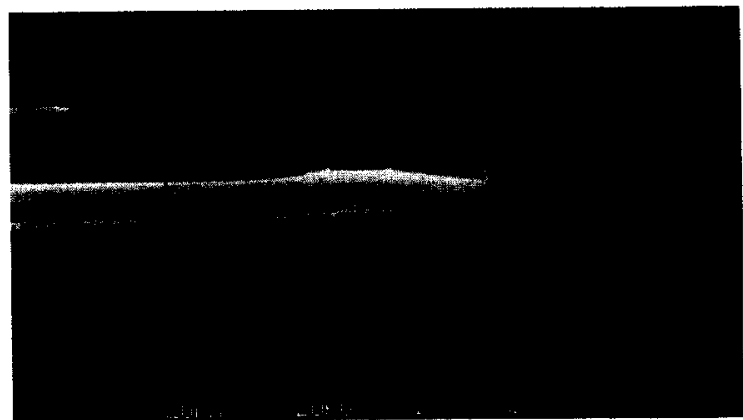

Fig. 6. Close-up of the capacitive microprobe head.

trolled contact pressure. Subsequent high-temperature annealing $\left(1100^{\circ} \mathrm{C}, 3 \mathrm{~h}\right)$ seals the two wafers on the selected surfaces together (Fig. 5(b)). Bulk etching in potassium hydroxide (KOH: $40 \%, 60{ }^{\circ} \mathrm{C}$ ) is time controlled and stopped when a $20 \mu \mathrm{m}$ membrane is left (Fig. 5(c)). Then a $1 \mu \mathrm{m}$ thick thermal $\mathrm{SiO}_{2}$ is grown and patterned to form the mask for the tip formation in RIE (Fig. 5(d)). Wet etching in $\mathrm{HNO}_{3}: \mathrm{HF}: \mathrm{CH}_{3} \mathrm{COOH}$ releases the cantilever and counter electrode from the membrane and forms a high protruding silicon tip (Fig. 5(e)). By selective BHF etching of the sacrificial $\mathrm{SiO}_{2}$ spacer between the two bonded beams, we obtain the micromechanical air-gap capacitor with a gap of $3 \mu \mathrm{m}$ (Fig. 5(f)), which is defined by the thickness of the bonding interface layer (1.5 $\mu \mathrm{m} \mathrm{SiO}{ }_{2}$ on both wafers). Gap distances of 2 or $1 \mu \mathrm{m}$ are feasible by using thinner bonding dioxide layers. The individual sensors are attached by thin silicon beams to the wafer-chip and can easily be removed. Figure 6 shows a close-up of a micromachined microlever head.

\section{Results and discussion}

The microfabricated devices were encapsulated and wire-bonded for testing. A total capacitance of $\approx 3 \mathrm{pF}$ between the connection pads has been measured. When applying bias voltages the cantilever motion could be observed with an optical confocal microscope. Figure 7 shows both the measured and calculated deflections of one cantilever as a function of the applied d.c. voltage. The measured values show the expected $V^{2}$ behaviour and are, for small deflections, in good agreement with the theoretical curve. A spring constant of $k=5 \mathrm{~N} / \mathrm{m}$ has been assumed here, meaning a somewhat stiffer cantilever than expected. A possible explanation for this could be a slightly thicker cantilever as designed and the additional aluminium layer of $0.5 \mu \mathrm{m}$ required for low ohmic contacting of the bonding wires.

Figure 8 shows the measured amplitude of vibration for a cantilever with $l \approx 300 \mu \mathrm{m}$ and $w=40 \mu \mathrm{m}$ when

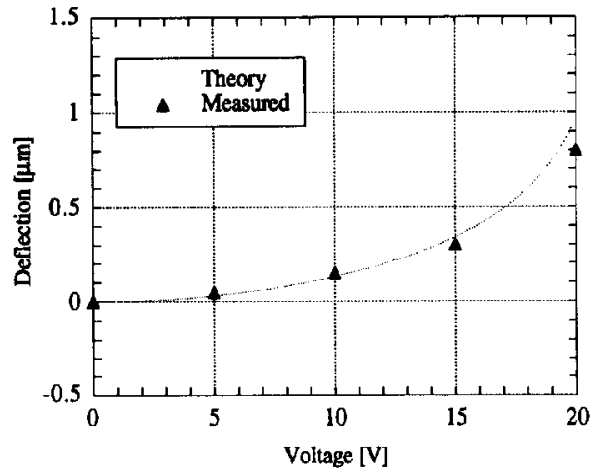

Fig. 7. Static deflection curve of a microlever as a function of applied d.c. bias voltage. The measured values (confocal microscope) and the theoretical curve $(k=5 \mathrm{~N} / \mathrm{m})$ are presented.

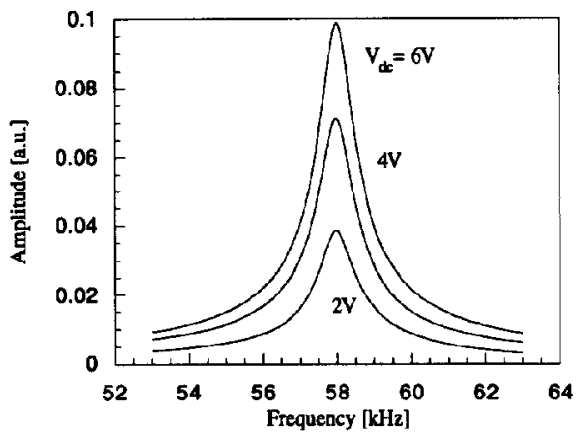

Fig. 8. Measured amplitude of vibration of the electrostatically actuated cantilever, $V_{\mathrm{ac}}=1 \mathrm{~V}$ peak to peak; $V_{\mathrm{dc}}=2,4,6 \mathrm{~V} ; l=300$ $\mu \mathrm{m}, w=40 \mu \mathrm{m}$.

the frequency of the harmonic excitation voltage of 1 $V$ (peak to peak) is swept from 53 to $63 \mathrm{kHz}$. The measurements were performed in air with a confocal microscope and a phase-sensitive detection of its output signal (lock-in amplifier). The amplitude $z$ of vibration is observed to increase almost linearly with applied d.c. voltage (Fig. 8) and a.c. voltage (not shown), as predicted by eqn. (14). A resonance frequency of $58 \mathrm{kHz}$ and a quality factor of $Q=\omega / \Delta \omega=65$ are measured, where $\Delta \omega$ is the full bandwidth at 0.707 of the maximum amplitude [11]. At resonance the observed amplitude of vibration is about $30 \mathrm{~nm}$ for a d.c. voltage of $6 \mathrm{~V}$. Equations (1), (2), (10) and the measured resonance frequency of $58 \mathrm{kHz}$ yield a spring constant $k=3.26$ $\mathrm{N} / \mathrm{m}$ and a voltage $V_{\mathrm{t}}=35.5 \mathrm{~V}$. From eqn. (12) we thus compute a lowering of the resonance frequency to 57.75 $\mathrm{kHz}$ at $V_{\mathrm{dc}}=6 \mathrm{~V}$. This expected lowering with increasing applied d.c. voltage is not observed. The discrepancy is probably due to uncertainties in the cantilever dimensions and also to the limited range of voltages used during the experiments. 


\section{Conclusions}

We have demonstrated the feasibility of microfabricated capacitive microlevers. Theoretical modelling and preliminary measurement results illustrate their potential use in scanning force microscopy.

\section{Acknowledgements}

The authors wish to acknowledge V.P. Jaecklin and C. Linder from the IMT and M. Binggeli and R. Christoph from the CSEM for helpful discussions. Technical assistance from P.A. Clerc, S. Jeanneret and S. Pochon from the IMT is gratefully acknowledged. This work is financially supported by the Swiss National Science Foundation.

\section{References}

1 G. Binnig, C. Quate and Ch. Gerber, Atomic force microscope, Phys. Rev. Lett., 56 (1986) 930.

2 For example, D. Rugar and P. Hansma, Atomic force microscopy, Physics Today, (Oct.) (1990) 23-30.

3 M. Tortonese, H. Yamada, R.C. Barrett and C.F. Quate, Atomic force microscopy using a piezoresistive cantilever, Tech. Digest, 6th Int. Conf. Solid-State Sensors and Actuators (Transducers '91), San Francisco, CA, USA, June 24-28, 1991, pp. 448-451.

4 T. Itoh and T. Suga, Piezoelectric force sensor for scanning force microscopy, Tech. Digest, 7th Int. Conf. Solid-State Sensors and Actuators (Transducers '93), Yokohama, Japan, June 7-10, 1993, pp. $610-613$.

S S. Akamine, T.R. Albrecht, M.J. Zdeblick and C.F. Quate, A planar process for microfabrication of a scanning tunneling microscope, Sensors and Actuators, A21-A23 (1990) 964-970.

6 L.C. Kong, B.G. Orr and K.D. Wise, A micromachined silicon scan tip for an atomic force microscope, IEEE Solid-State Sensor and Actuator Workshop, Hitton Head Island, SC, USA, 1990, pp. 28-31.

7 T. Göddenhenrich, H. Lemke, U. Hartmann and C. Heiden, Force microscope with capacitive displacement detection, $J$. Vac. Sci. Technol., A8 (1) (1990) 383-387.

8 G. Neubauer, S.R. Cohen, G.M. McClelland, D. Horne and C.M. Mate, Force microscopy with a bidirectional capacitance sensor, Rev. Sci. Instrum., 61 (9) (1990) 2296-2308.

9 G. Binnig, U. Dürig, J.K. Gimzewski, D. Pohl and H. Rohrer, Atomic force sensor head, Eur. Patent Appl EP-A 0290648 (May 12, 1987).

10 R.A. Buser and N.F. de Rooij, Very high $Q$-factor resonators in monocrystalline silicon, Sensors and Actuators, A21-A23 (1990) 323-327.

11 D. Sarid, Scanning Force Microscopy, Oxford University Press, New York, 1991.

12 R.J. Roark and W.C. Young, Formulas for Stress and Strain, McGraw-Hill, Singapore, 5th edn., 1976, Ch. 7.

13 H.C. Nathanson, W.E. Newell, R.A. Wickstrom and J.R. Davis, Jr., The resonant gate transistor, IEEE Trans. Electron Devices, ED-14 (1967) 117-133.
14 C. Linder, Electromechanical polysilicon structures and micromachining processes for sensor and actuator applications, Ph.D. Thesis, University of Neuchâtel, IMT, 1993.

15 S. Timoshenko, D.H. Young and W. Weaver, Vibration problems in Engineering. Wiley, New York, 4th edn., 1974, pp. 72-77.

16 J. Brugger, R.A. Buser and N.F, de Rooij, Silicon cantilevers and tips for scanning force microscopy, Sensors and Actuators A, 34 (1992) 193-200.

\section{Biographies}

Jürgen Brugger received his M.Sc. degree in physicalelectronics in 1989 from the University of Neuchâtel in Switzerland. He is involved in research work for his Ph.D. degree at the Institute of Microtechnology (IMT) Neuchâtel, in the area of silicon microstructures for scanning force microscopes. He is currently working at the Central Research Laboratory, Hitachi Ltd., in Japan.

Nicolas Blanc received his diploma in physics from the Swiss Federal Institute of Technology in Lausanne in 1988 and his Ph.D. degree from the same institute in 1992. The thesis work was done at the IBM Zurich Research Laboratory, Switzerland, and focused on electronic transport phenomena in GaAs/AlGaAs semiconductor nanostructures. $\mathrm{He}$ is currently project leader at the IMT, Neuchâtel, in the field of silicon microstructures applied to scanning force microscopy and resonant micromechanical devices.

Philippe Renaud received his diploma in physics from the University of Neuchâtel, Switzerland, in 1983 and his Ph.D. degree from the University of Lausanne, Switzerland, in 1988. He stayed as a postdoctorate researcher at Berkeley University, CA, and then at the IBM Zurich Research Laboratory in Switzerland to develop scanning tunnelling microscopes for low temperature and luminescence studies, respectively. Since 1992 he has worked on sensors and actuators in the Swiss Center for Electronics and Microtechnology (CSEM), Neuchâtel. He is now also assistant professor at the Swiss Federal Institute of Technology in Lausanne.

Nicolaas $F$. de Rooij received the M.Sc. degree in physical chemistry from the State University of Utrecht, Netherlands, in 1975, and the Ph.D. degree from Twente University of Technology, Netherlands, in 1978. From 1978 to 1982 , he worked at the Research and Development Department of Cordis Europa N.V., Netherlands. In 1982, he joined the IMT as professor and head of the Microfabricated Transducers Group. Since October 1990, he has been acting as director of the IMT. Since 1987, he has been a lecturer at the Swiss Federal Institute of Technology, Zürich (ETHZ), and since 1989 , he has also been a professor at the Swiss 
Federal Institute of Technology, Lausanne (EPFL). His research activities include microfabricated sensors and actuators. He is a member of the steering committee of the International Conference on Solid-State Sensors and Actuators and of Eurosensors. He acted a European
Program Chairman of Transducers '87 and General Chairman of Transducers ' 89 . He is a member of the editorial board for the journals Sensors and Actuators, Sensors and Materials and Joumal of Microelectromechanical Systems. 